\title{
CAPITAL HUMANO, CALIDAD EDUCATIVA Y CRECIMIENTO ECONÓMICO
}

Silvina Elías*

\section{Resumen}

En la reciente literatura acerca del crecimiento económicose ha asignadoespecialénfasisalaformación de capital humano en el crecimiento económico de las naciones.El objetivo del trabajo es profundizar la investigación de la importancia de la calidad en la formación del capital humano y su influencia en el crecimiento económico. A tal fin, se realizará un análisis de panel de datos y uno de cross-section en el período 1960-2000 para una muestra de países, clasificandólos de acuerdo a las diferencias en los niveles de ingreso. Es que se intenta evaluar si la calidad del capital humano como factor explicativo del crecimiento varía en los distintos países según los niveles de ingreso per cápita.

Palabras clave:capital humano - crecimiento económico - calidad educativa

\section{Abstract}

Recent literature about economic growth has assigned special emphasis to human capital formation in the economic growth of nations. This paper focuses on human capital quality as a determinant of economic growth. The analysis stresses the distinction between the quantity and the quality of education. It is a cross section and a panel data study for a group of international countries. The period of the empirical study is 1960-2000 and countries are classified taking into account differences in their income per capita levels.

Keywords: human capital - economic growth - educational quality

\section{INTRODUCCION}

Durante fines de los ochenta y comienzos de los noventa las nuevas teorías sobre el crecimiento económico enfatizaron la posición del capital humano al analizar las diferencias internacionales en la tasa de crecimiento económico de los países (Lucas (1988), Romer $(1986,1990))$. La teoría "neoclásica” convencional del crecimiento sostenía que el crecimiento económico era el resultado de la acumulación de capital físico y de la ampliación de la fuerza de trabajo, combinadas con un factor exógeno, el progreso tecnológico, que incrementaba la productividad del capital y de la fuerza del trabajo. Según la nueva teoría del crecimiento, lo que aumenta la productividad no es un factor exógeno sino factores "endógenos", relacionados con la acumulación de los factores de producción y su nivel de conocimientos.

Los modelos de capital humano demuestran la forma en que la educación posibilita que todo el proceso de producción se beneficie con las externalidades que una sociedad más educada genera. La mano de obra más capacitada utiliza el capital de manera más eficiente, con lo cual pasa a ser más productiva. Es también más probable que se introduzcan innovaciones de modo de idear nuevas y mejores formas de producción. Más aún, la difusión de los beneficios de la mano de obra capacitada aumenta la eficiencia global del trabajo. De esta manera, la elevación del nivel de educación provoca un aumento de la eficiencia de todos los factores de producción.

La cuestión fundamental es el alcance de la inversión en capital humano para contribuir al crecimiento económico. A nivel microeconómico, uno de los resultados más recuentes de la 
literatura es que la formación de capital humano, considerado como mejoras en la ducación, tiende a aumentar los salarios. Comenzando con el trabajo original de Mincer (1974), numerosos estudios (Card y Krueger (1992), Klenow y Rodriguez-Clare (1997), Hall y Jones (1998)) han estimado una ecuación en la cual la tasa de salario de un individuo se regresa en función de los años de escolaridad y experiencia (comúnmente conocida como ecuación de salarios de Mincer). Estos trabajos han encontrado estimaciones significativas de los retornos de la educación para una diversidad de países en diferentes etapas de desarrollo. En el ámbito macroeconómico, la evidencia es variada. La mayoría de los estudios empíricos de crecimiento económico de largo plazo incluyen alguna variable proxy del capital humano. Las limitaciones en los datos han sido una restricción importante en el momento de realizar prescripciones de política económica. Los trabajos de Barro (1991), Mankiw, Romer y Weil (1992), entre otros, utilizan las tasas de escolaridad y encuentran una contribución positiva y significativa del capital humano al crecimiento del producto. Por otro lado, Kyriacou (1991), Lau y otros (1991) y Benhabib y Spiegel (1994), identifican una relación no significativa e incluso negativa para el stock de capital humano (medido como la media de años de educación alcanzada). Como se analizará más adelante, el efecto estimado del capital humano varía según la forma en que es medido (como stock o como flujo) ${ }^{1}$. Es necesario entonces tener en claro cuál es el potencial explicativo del capital humano en el crecimiento económico al tomar decisiones de política macroeconómica. En general, las variables que se utilizan como proxies del capital humano (tasas de escolaridad o el promedio de años de educación adquirido) en los estudios empíricos son medidas imperfectas del componente educacional del capital humano. Estas miden la cantidad pero no la calidad de la educación y por lo tanto no toman en cuenta limitaciones de estructura y funcionamiento del sistema educativo que obstaculizan un mejor aprovechamiento del capital humano. En este contexto, el objetivo del presente trabajo es profundizar la investigación acerca de la importancia de la calidad en la formación del capital humano y su influencia en el crecimiento económico a través de un estudio empírico.

Aunque el capital humano incluye no solo a la educación, sino también a la salud y aspectos del "capital social”, el foco principal de este trabajo es la educación y la calidad de la misma y sus efectos sobre el crecimiento económico de los países. A tal fin, se realizará un análisis de panel de datos y uno de cross-section en el período 1960-2000 para una muestra de varios países clasificándolos de acuerdo a las diferencias en los niveles de ingreso. Es aquí que se intenta evaluar si la calidad del capital humano como factor explicativo del crecimiento económico varía en países con diferentes niveles de ingreso per cápita.

En la próxima sección se analiza la relación entre capital humano y crecimiento económico, con especial énfasis en la contribución de la educación como un componente medible del capital humano. Dentro del nivel empírico se identifican los problemas de medición que pueden surgir al buscar las variables proxies del capital humano. Al final de la sección se profundiza la importancia de la calidad en el estudio del capital humano. En la sección tercera se presenta el análisis de un modelo de crecimiento que incluye no solo la cantidad sino también la calidad del capital humano. Se realiza el estudio empírico con las variables y una muestra de países seleccionados.

\section{CRECIMIENTO Y CAPITAL HUMANO}

La introducción del capital humano en la función de producción puede evitar la restricción de rendimientos decrecientes hacia un concepto más amplio del capital y lograr el crecimiento per cápita de largo plazo en ausencia del cambio tecnológico exógeno. De aquí que el capital humano pueda ser una alternativa para explicar el crecimiento. En este marco, se lo considera como el potencial generador de renta personal. Comprende la capacidad y talento innatos, así como la

\footnotetext{
* Departamento de Economía, Universidad Nacional del Sur.

${ }^{1}$ Los primeros estudios utilizaban medidas de flujo (tasas de escolaridad), mientras que los estudios más recientes han utilizado una medida de stock (promedio de años de educación derivado de la acumulación de las tasas de escolaridad pasadas).
} 
educación y cualificaciones adquiridas. El capital humano se adquiere por medio de la educación formal dada por el sistema educativo y la formación de carácter informal adquirida de la experiencia en el trabajo. ${ }^{2}$

\section{I.1.- Los fundamentos teóricos}

Para Aghion y Howitt (1998) dentro de la literatura del crecimiento endógeno se pueden diferenciar dos tipos de modelos que analizan la relación entre educación y crecimiento. Los primeros iniciados por Lucas (1988) e inspirados en la teoría del capital humano de Becker, están basados en la idea de la acumulación de capital humano. En este marco el proceso de acumulación de capital humano es congruente al del capital físico: es costoso y sustrae tiempo a la producción pero representa una inversión rentable. De esta forma, las diferencias en las tasas de crecimiento entre países son atribuibles a diferencias en las tasas de crecimiento a la que los países acumulan capital a lo largo del tiempo. El segundo grupo de modelos, que se remonta a las contribuciones de Nelson y Phelps (1966) y que ha sido retomado por la literatura schumpeteriana del crecimiento (Romer (1990)), describe el crecimiento originado por el stock de capital humano, lo que afecta la habilidad de un país para innovar y alcanzar a los países más avanzados. Estos modelos consideran que el capital humano acumulado a través del proceso productivo es un requisito para el desarrollo de las aptitudes necesarias para desarrollar y adaptar el cambio tecnológico. De acuerdo a esta literatura, las diferencias en las tasas de crecimiento entre los países son debidas, en primer lugar, a diferencias en los stocks de capital humano y surgen así diferencias de acuerdo a las habilidades de los países para generar el progreso técnico. El capital humano se convierte entonces en un "input" fundamental para aprender y entender nuevos desarrollos tecnológicos, así como para la generación de nuevas ideas. Estos modelos pueden explicar la $\beta$ convergencia, pero ésta no es causada por la existencia de rendimientos decrecientes sino por las transferencias de tecnología. En este marco se espera que cuanto mayor sea el nivel de capital humano y la brecha de tecnología entre los “seguidores” y el "líder” 3 , mayor será la tasa de crecimiento resultante.

Gary Becker (1964) fue el pionero en desarrollar la teoría del capital humano. Examina los determinantes de los ingresos y del bienestar a través de la acumulación de conocimientos y habilidades. La educación formal en las escuelas es un ejemplo de adquisición de habilidad. A su vez, las inversiones en entrenamiento laboral es otro ejemplo de acumulación de capital humano. De este modo el modelo provee explicaciones convincentes sobre porqué algunos trabajadores ganan más que otros. A medida que los trabajadores adquieren mayores habilidades a través de las inversiones en capital humano, ganan mayores ingresos al aumentar su productividad.

Según Becker pueden considerarse dos tipos de formación en el puesto de trabajo, la general y la específica. La primera le es útil a muchas empresas y no sólo a las que la proporcionan. En principio, prácticamente toda la formación en el trabajo da lugar a incrementos de la productividad marginal futura de los trabajadores en las empresas que la proporciona; sin embargo, cuando la formación es general, también se incrementa el producto marginal en otras empresas. En un mercado laboral competitivo, los salarios que paga cualquier empresa dependen de las productividades marginales en otras empresas, los salarios y los productos marginales futuros aumentarían en las empresas que proporcionan formación de tipo general. La formación perfectamente general sería igualmente útil en muchas empresas, y los productos marginales aumentarían por igual en todas ellas. Por lo tanto, los salarios podrían elevarse exactamente en la misma medida que el producto marginal, y las empresas que proporcionasen ese tipo de formación no podrían obtener parte alguna de su rendimiento. Las empresas sólo proporcionan formación general si no incurren en coste alguno. Los individuos que reciben formación general estarán dispuestos a costearla ya que la formación hace que aumenten sus salarios futuros. Son, por lo

\footnotetext{
${ }^{2}$ A los efectos de este trabajo se dará énfasis a la incorporación de capital humano dada por el sistema de educación formal y no se contemplará un concepto más amplio del mismo que involucra el status nutricional y sanitario de la fuerza de trabajo, etc.

${ }^{3}$ Una definición más explícita de “líder” y “seguidor” puede encontrarse en Benhabib y Spiegel (1994).
} 
tanto, los aprendices, y no las empresas, los que pagan por la formación general, y los que perciben su rendimiento.

Por otra parte la formación específica es aquella que eleva la productividad en mayor medida en las empresas que la proporcionan. La formación totalmente específica es aquella que afecta a la productividad de las personas formadas, pero solamente en la empresa que la proporciona. La mayor parte de la formación que se imparte en el trabajo no es ni completamente general ni completamente específica, pero eleva la productividad en mayor grado en las empresas que la proporcionan $\mathrm{y}$, por lo tanto, debe considerarse como formación específica. El resto de la formación eleva la productividad en la misma medida en todas las empresas y, por lo tanto, debe considerarse como formación general.

Inspirado en la teoría del capital humano de Becker, Lucas (1988) considera una economía conformada por individuos de vida infinita que eligen en cada momento cómo asignar su tiempo entre producción corriente y adquisición de habilidades (schooling) donde esta última aumenta la productividad en los períodos futuros. Incorpora al modelo AK de capital físico una externalidad en el capital humano. La inversión individual en educación no sólo incrementa su propia productividad, sino que genera también un efecto externo al extender la base de conocimientos generales de la sociedad, contribuyendo a elevar la productividad del resto de los trabajadores y el nivel de producto. Así entonces:

$$
y=k^{\beta}(\mu h)^{1-\beta}
$$

donde:

$\mathrm{k}=$ stock de capital físico

$\mathrm{h}=$ stock de capital humano del agente representativo

$\mu=$ fracción de tiempo destinada a la producción

La siguiente ecuación muestra cómo el tiempo de escolarización afecta la acumulación de capital humano. En contraste con la teoría de la innovación, la adquisición de capital humano no incorpora externalidades o spillovers entre individuos de la misma generación.

$$
\dot{h}=\delta h(1-\mu) \delta>0
$$

donde

$\dot{h}=$ tasa de aacumulación de capital humano

1 - $\mu$ = tiempo de escolarización

$\delta$ = parámetro de productividad

Si $\mu=1$, no hay formación de capital humano. Si $\mu=0$, todo el esfuerzo se destina a acumular capital humano que crece a la tasa máxima posible, $\delta$. Entre estos extremos no hay rendimientos decrecientes al capital humano, un aumento de un porcentaje dado de $h$ siempre requiere el mismo esfuerzo independientemente del nivel alcanzado. Esto parece desafiar la observación empírica del patrón individual de acumulación de capital humano; se aprende mucho más en los primeros años de vida, luego se aprende menos rápidamente y finalmente nada. Una explicación alternativa a esta observación es que la vida finita de una persona hace a la inversión en capital humano tanto menos rentable cuanto más tardía es. Para salvar esto Lucas supone individuos de vida infinita, se trata más bien de una dinastía que de un solo individuo y donde los conocimientos se transmiten de generación en generación. Cada nuevo miembro comienza con un nivel inicial de capital humano proporcional al nivel alcanzado por los miembros más viejos de la 
dinastía. De esta manera la acumulación de capital humano es una actividad social que involucra grupos de personas en una forma que no tiene contrapartida en la acumulación de capital físico.

El supuesto de que la acumulación de capital humano incluye rendimientos constantes a escala al stock de capital humano existente produce una tasa de crecimiento positivo en el steady state igual a:

$g=\delta\left(1-\mu^{*}\right)$

donde

$\mu^{*}=$ óptima asignación de tiempo entre producción

y educación

$\mu$ * maximice la función de utilidad intertemporal

del consumidor representativo

$U=\int_{0}^{\infty} \frac{C_{t}^{1-\sigma}}{1-\sigma} e^{-\rho t}$

sujeto a (1), (2) y $\dot{k}=\mathrm{y}-\mathrm{c}$

El esfuerzo en educación puede depender negativamente de la tasa de preferencia intertemporal y del coeficiente de aversión al riesgo y positivamente de la productividad de la escolarización. La principal originalidad del modelo reside en la explicación de las disparidades internacionales que se desprende del análisis dinámico. Desde cualquier posición inicial, con dotaciones dadas de capital físico y humano, la economía converge a la trayectoria del crecimiento equilibrado pero no a un único punto de equilibrio sino a distintos puntos cuya ubicación va a depender de la situación inicial. Así, una economía con dotación inicial baja de ambos tipos de capital experimentará un crecimiento menor a otra economía mejor dotada y permanecerá por debajo de esta última. El trabajo será también mejor remunerado en el país con mayor nivel de capital humano porque la productividad del mismo se ve incrementada por el efecto de la externalidad.

El modelo de Lucas es elegante y simple pero carece de realismo. Por ejemplo, la ecuación de acumulación del capital humano $(\dot{h})$ indica que los retornos de la educación del individuo permanecen constantes a lo largo de toda su vida. Este supuesto está en desacuerdo con la evidencia empírica en educación y con la teoría del capital humano de Becker, quien sugiere que los retornos de la educación tienden a disminuir durante la vida del individuo. En los hechos se puede observar que se aprende mucho al principio, lo que daría una variación porcentual de $h$ menor que uno.

Una forma simple de reformular el modelo de Lucas salvando esta objeción sería considerar un marco de generaciones superpuestas donde los individuos heredan el capital humano acumulado por sus padres. En este sentido han contribuido Azariadis y Drazen (1990), al considerar un modelo de generaciones superpuestas con trampas de bajo crecimiento. Analizan un modelo donde hay una generación continua de familias superpuestas donde cada individuo vive dos períodos. Todos los individuos que nacen en el momento t heredan el capital humano agregado acumulado por la generación previa nacida en el período t-1. Si el stock inicial de capital humano heredado de las generaciones precedentes es débil, entonces la eficacia del aprendizaje será baja. Si ésta es inferior al rendimiento del capital físico, entonces los individuos prefieren acumular capital físico y trabajar en lugar de acumular capital humano. La economía se encuentra entonces en una situación de estancamiento del capital humano y del producto. Este sendero de bajo crecimiento o 
trampas de bajo desarrollo puede coexistir naturalmente con un sendero de alto crecimiento donde todas las generaciones invierten por lo menos el nivel mínimo en educación. Este es el caso más interesante considerado por Azariadis y Drazen, donde la tecnología educativa despliega externalidades positivas. La existencia de externalidades con umbrales en la tecnología educativa puede llevar a múltiples senderos de crecimiento, incluyendo una trampa de bajo crecimiento donde la insuficiente inversión en educación en el pasado desalienta la adquisición de habilidades en el futuro crecimiento. De esta forma Azariadis y Drazen proveen una explicación más "natural" que la de Lucas referida a la razón de que los países con distintas dotaciones de capital humano inicial pueden seguir creciendo a distintas tasas. También sugieren el rol de la intervención del estado en el sector educativo para evitar las trampas de bajo crecimiento y promover el crecimiento sostenido.

Romer (1990), a diferencia de Lucas que introduce al capital humano como un factor de producción de bienes finales, otorga al capital humano un rol en la producción de tecnología. Tiene por lo tanto un efecto sobre la tasa de crecimiento del progreso técnico, es decir que un aumento en el stock de capital humano conducirá en este modelo a una aceleración del crecimiento debido a un aumento en la Investigación y Desarrollo. Como ya fuera sugerido por Nelson y Phelps (1966), un mayor stock de capital humano permite a un país absorber con mayor facilidad las nuevas ideas y productos que han sido descubiertos en países más avanzados. De esta forma un país seguidor con mayor dotación de capital humano tenderá a crecer más rápido porque incorpora más velozmente la tecnología de los líderes.

Romer realiza una importante distinción entre capital humano y conocimiento; el primero es la capacidad creativa del individuo y se encarna en su persona. De esta forma el capital humano es un bien económico convencional que desaparece cuando la persona muere; es el componente rival del conocimiento que tiene en cuenta el nivel de educación y la experiencia. En cambio, el conocimiento creado por una persona es un intangible no incorporado a ningún objeto físico y sobrevive a la persona.

Laing, Palivos y Wang (1995) examinan la tasa de crecimiento endógeo de una economía en la cual las fricciones de corto plazo de los mercados son una parte del ambiente económico. La utilización de un marco de búsqueda y contrato permite relajar el supuesto de un consumidor productor integrado, permitiendo explicitar el análisis de la interacción entre las firmas y los trabajadores. Siguen a Romer $(1986,1989)$, Lucas (1988) y Stokey (1991), al asumir que la acumulación de capital humano es el primer motor de crecimiento económico. Los trabajadores acumulan una proporción del stock de conocimiento público al invertir en educación cuando son jóvenes. Suponen también que los esfuerzos de escolarización de los trabajadores tienen influencia tanto en su productividad inicial como en la subsiguiente tasa de crecimiento una vez que están empleados. Cuando los individuos ya están escolarizados comienzan su búsqueda de empleo. Demuestran, entre otras cosas, que una mejora en la eficacia de ensamble ("matching efficiency") promueve el crecimiento económico mediante incrementos en los retornos de la educación.

En síntesis, las teorías de crecimiento endógeno, donde el comportamiento del capital humano depende de la educación formal, proveen un marco analítico en el que esta variable juega un papel importante como fuente del crecimiento económico. En particular, se pueden distinguir los efectos del nivel del capital humano y de la acumulación del capital humano (es decir cuánto está creciendo el nivel de educación en el tiempo).

Hay dos líneas de pensamiento que pueden asociarse a los trabajos seminales de Lucas (1988) y Nelson y Phelps (1966) respectivamente. De acuerdo con los modelos que siguen a Lucas, el nivel de producto depende del nivel de capital humano, pues éste es un input como cualquier otro dentro de la función de producción. Concluyen que la tasa de crecimiento del producto depende de la tasa de crecimiento del capital humano por lo que solo es posible conseguir más output si se adquiere más input. Por el contrario, en el enfoque de Nelson y Phelps, el capital humano no es un input como cualquier otro sino que es la primera fuente de innovaciones. Por lo tanto, la tasa de 
crecimiento del producto va a depender de la tasa de innovación. Del nivel más que de la tasa de crecimiento del capital humano. Según Topel (1999), es plausible que ambas teorías sobre el rol del capital humano sean ciertas. El crecimiento del capital humano puede incrementar el output y establecer la base para el crecimiento futuro. Para este autor, las diferencias entre dichas teorías es más semántica que real pues los autores neoclásicos definen al capital humano con mayor amplitud incluyendo en el mismo la acumulación de conocimientos y habilidades para aplicar en procesos productivos.

Si bien estas teorías han tenido una gran aceptación entre los economistas durante los últimos años, no se debe dejar de aclarar que existe también otra línea de trabajos dentro de la literatura, que utilizan la hipótesis planteada inicialmente por Arrow (1962) del "aprendizaje en el trabajo" ("learning by doing"). De acuerdo con esta idea, en ciertas industrias se producirá un fuerte aumento en la productividad global al expandirse los volúmenes de producción. Una expresión de estos aumentos de productividad es la aparición de nuevos y más sofisticados bienes como consecuencia del aprendizaje que trae consigo el incremento del volumen de producción (Romer (1994)).

\section{I.2.-Evidencia empírica}

Como se ha indicado en la sección anterior, los recientes modelos teóricos sugieren que el nivel de educación adquirido es un importante determinante del crecimiento económico. En esta sección se revisará la evidencia para intentar cuantificar estos efectos. Para Topel (1999) existen dos corrientes principales de la investigación empírica en el crecimiento económico. Ambas intentan medir el efecto de las diferencias en los inputs sobre la productividad y el ingreso per cápita. Por un lado el "growth accounting", que divide el crecimiento del producto entre los cambios en aquellos inputs cuantificables -capital físico y humano- y un residuo llamado productividad total del factor (TFP). Asumiendo rendimientos constantes de escala y mercado de factores en condiciones de competencia, la tasa de cambio del producto para el país $i$ en el momento $t$ está dada por la siguiente ecuación:

$$
\dot{y}_{i t}=\alpha_{i t} \dot{k}_{i t}+\left(1-\alpha_{i t}\right) \dot{h}_{i t}+\dot{p}_{i t}
$$

donde

$\dot{y}, \dot{k}, \dot{h}, \dot{p}$

representan las tasas de cambio proporcional del producto, del capital físico, del capital humano y TFP respectivamente, y donde $\alpha$ representa la participación del capital en el ingreso nacional. Con excepción de $p$, todas las magnitudes de la ecuación son medibles, lo que deja a la TFP como la porción del crecimiento del producto que permanece inexplicado luego de tomar en cuenta las tasas de crecimiento del capital físico y humano. De hecho, la estimación de TFP es comúnmente llamada residuo de Solow.

Dentro de las más recientes aplicaciones de este método se encuentran los trabajos de Young $(1992,1994,1995)$, quien examina y compara la perfomance de crecimiento de cuatro economías del sudeste asiático (Corea del Sur, Hong Kong, Taiwan y Singapur). Encuentra que el mejor nivel de educación adquirido por la fuerza laboral incrementa la tasa de crecimiento anual del effective labour input en aproximadamente un 1\% (Young 1995,p.645). Antes de los trabajos de Young, muchos observadores atribuían el crecimiento récord de estas economías a mejoras tecnológicas, derivadas posiblemente de políticas gubernamentales que favorecían el crecimiento de ciertas industrias y tecnologías. Midiendo cuidadosamente las cantidades de capital físico y humano de estos países, Young concluye que su rápido crecimiento se debe a la acumulación de los factores (y utilización en el caso del trabajo), mientras que el crecimiento de la TFP no es tan alto como corroboran otros estudios. El principal inconveniente de este enfoque radica en la dificultad de medir los inputs especialmente cuando es algo intangible como el capital humano. 
Otra limitación de estos modelos es que son meramente descriptivos y no tienen en cuenta cómo se realiza la acumulación de los factores o si la acumulación de capital humano es esencial para el crecimiento.

La otra corriente de trabajos citada por Topel (1999) se orienta a la utilización de regresiones, estimando modelos cross-section y de panel de datos que analizan los determinantes del ingreso de los países. Debido a que el principal interés de este trabajo es determinar qué conclusión se puede extraer de la relación empírica entre educación y crecimiento económico; en esta sección se abordará especialmente la segunda corriente de trabajos citada por Topel.

A principios de los noventa aparecieron numerosos trabajos empíricos con estimaciones de modelos de crecimiento incorporando el capital humano, utilizando datos cross-section. Esta evidencia empírica se inspira principalmente en las teorías de crecimiento endógeno. Sin embargo, el marco para el trabajo aplicado obedece también al viejo modelo neoclásico que fuera desarrollado en los '50 y '60. Los recientes estudios empíricos combinan esquemas del modelo neoclásico, especialmente el concepto de convergencia por el cual las economías más pobres tienden a alcanzar a las más ricas- con extensiones que enfatizan el rol de las políticas gubernamentales y de las instituciones.

Los recientes modelos de crecimiento endógeno son útiles para entender porqué las economías pueden continuar creciendo en el largo plazo no obstante la existencia de los rendimientos decrecientes en la acumulación de capital físico y humano. En contraste, el marco del modelo neoclásico extendido sirve como vehículo para interpretar las tasas de crecimiento relativo entre los países y explicar por qué, por ejemplo, un país como Corea del Sur creció mucho más que Estados Unidos en los últimos treinta años. En efecto, las nuevas y viejas teorías son complementarias y no compiten entre sí (Barro (1998)).

Con el objeto de hacer compatibles las predicciones del modelo de crecimiento neoclásico con la evidencia empírica, Mankiw, Romer y Weil (1992) presentaron un modelo neoclásico ampliado, al adoptar una definición más amplia de capital, incluyendo dentro del stock de capital al capital humano. Este modelo considera en el capital otras formas de capital distinto al capital físico (capital privado, infraestructuras, equipamientos sociales, etc.). El modelo incluye el conjunto de conocimientos de los trabajadores adquiridos por medio del sistema educativo y en su curva de aprendizaje -learning by doing-, y que forman un stock de habilidades y recursos que inciden directamente sobre los niveles de eficiencia de la economía. Mediante una función de producción Cobb-Douglas, el modelo de Mankiw, Romer y Weil (1992) puede expresarse de la siguiente forma:

$$
Y=K^{\alpha} H^{\beta}(A L)^{1-\alpha-\beta}
$$

donde:

$\mathrm{A}=$ nivel de la tecnología

$\mathrm{K}$ = stock de capital físico

$\mathrm{H}=$ stock de capital humano

$\mathrm{L}=$ número de trabajadores ocupados

$\alpha$ y $\beta=$ elasticidades del output respecto al capital físico y al capital humano.

El modelo presenta rendimientos constantes a escala y decrecientes sobre el capital físico. El análisis de la dinámica de esta economía es similar a la del modelo de Solow con la diferencia que ahora en lugar de considerar sólo la dinámica del capital físico también debe considerarse la del capital humano.

El capital humano se acumula de la siguiente forma: 


$$
H=s_{h} Y-\delta \dot{H}
$$

donde $s_{h}$ es la fracción de producto invertida en capital humano que es implementada empíricamente como la fracción de la población en edad de trabajar que se encuentra en nivel secundario en el período y $\delta$ es la tasa de depreciación del capital humano.

Este modelo, al incluir capital humano, predice efectos mucho mayores de cambios en los parámetros sobre el producto de lo que predice Solow. Las variaciones en los parámetros encontrados por MRW tienen el potencial de explicar alrededor de un $78 \%$ de las diferencias observadas en el producto per cápita para una muestra de países con datos correspondientes a 1985.

Benhabib y Spiegel (1994), siguiendo a los autores anteriores estiman una función de producción Cobb-Douglas donde el empleo y el capital físico y humano (stocks) se utilizan como factores de producción. Realizan estimaciones empíricas para el período 1965-1985. Encuentran que el logro en educación (como una medida del stock corriente de capital humano) permanece esencialmente sin correlación con el crecimiento si se utiliza un modelo Solow aumentado "a la Mankiw, Romer y Weil”, donde el capital humano no es más que un input ordinario de la función de producción. Sin embargo, el efecto del logro educativo se convierte en significativo si se sigue a Nelson y Phelps (1966).

El análisis de Benhabib y Spiegel es interesante no sólo porque provee soporte adicional a la teoría del crecimiento endógeno sino también porque sugiere que la divergencia en las tasas de acumulación de capital humano, como sugirió Lucas, se debe a diferencias en los stocks de capital humano que afectan la habilidad de los países más desarrollados para innovar la tecnología.

Por otra parte Barro (1991-1998) y Barro y Sala i Martín (1995) han explicado la tasa promedio de crecimiento en función de dos tipos de variables macroeconómicas: 1) niveles iniciales de variables estado (stock de capital físico y humano, entre otras), y 2) variables de control, las que reflejan las preferencias de agentes privados y gobierno como consumo del gobierno sobre el PBI, inversión doméstica sobre PBI, etc.. Como variables de educación utilizan los años de escolaridad promedio al comienzo del período para los distintos niveles de educación diferenciando por sexo y el gasto en educación como fracción del Producto Bruto Interno para el período 1965-1985 para una muestra de mas de 100 países. Sus principales conclusiones son que la educación alcanzada (medida como años promedio de escolaridad) está significativamente correlacionada con el crecimiento posterior aunque si se descompone la medida de educación agregada, el impacto de la educación primaria resulta insignificante y que el gasto público en educación también tiene un efecto positivo en el crecimiento.

Islam (1995) incorpora la técnica de panel de datos al análisis de crecimiento económico para salvar las diferencias que pueden existir en las funciones de producción de los distintos países. Utiliza el trabajo de Mankiw, Romer y Weil (1992) como punto de partida para luego establecer las diferencias que surgen con la utilización del panel de datos. Con respecto a las variables de capital humano, encuentran que la inclusión de la dimensión temporal en el análisis (dada por la técnica econométrica) destruye el efecto positivo que éstas tenían en el análisis de las regresiones de crecimiento.

La principal dificultad de los trabajos cross-country es que en una muestra de países muy heterogéneos no siempre se considera la importancia que tienen las diferencias en la naturaleza y calidad de la escolarización entre los distintos países. Intentando salvar esta dificultad existen algunos trabajos empíricos para muestras de países con características similares que corroboran las conclusiones de Barro y Sala i Martin. Por ejemplo J. Laitner (1993) contrasta el trabajo de Mankiw, Romer y Weil (1992) al analizar un modelo de crecimiento con capital físico y humano donde las decisiones de inversión para ambos tipos de capital son endógenas. En su modelo con 
rendimientos constantes de escala en la función de producción, la endogeneidad de ambos factores es lo que permite al crecimiento per cápita de largo plazo exceder la tasa del cambio tecnológico exógena. El capital humano es considerado como un bien privado, costoso de producir y que puede ser obtenido en diferentes cantidades. Realiza un análisis empírico para Estados Unidos, encontrando que la acumulación endógena de capital humano amplifica el efecto del progreso tecnológico exógeno en el crecimiento de largo plazo.

Englander y Gurney (1994) reestiman para los países OCDE regresiones de crecimiento basadas en el trabajo de Barro y Sala i Martin, entre otros, utilizando variables de capital humano como las tasas de escolaridad primaria y secundaria. Encuentran que para el período 1960-1980 el crecimiento del capital humano explica entre un $10 \%$ y un $20 \%$ del crecimiento del producto total.

De la Fuente y Da Rocha (1996) en una extensión del modelo de Mankiw, Romer y Weil, analizan para los países OCDE la influencia en la determinación de la tasa de progreso técnico de distintos indicadores del stock de capital humano y gasto en Investigación y desarrollo (I+D). El indicador de capital humano, que presenta un resultado más favorable, es la fracción de población con alguna educación superior, ya se incluya en la ecuación o no, con la variable asociada a la I+D como regresor adicional.

Gemmel (1996), también para los países OCDE, enfatiza los problemas de las tasas de escolaridad y construye medidas alternativas de capital humano basadas en la educación alcanzada en los niveles primario, secundario y terciario. Encuentra una correlación positiva entre el número de personas con educación superior y el crecimiento posterior del país. También concluye que la inversión en estos países está positivamente correlacionada con la extensión de la escolaridad secundaria en la fuerza laboral.

Elías y Fernández (1999) analizan regresiones de corte transversal para una muestra de 24 países latinoamericanos siguiendo el modelo planteado por Barro (1991, 1995). Utilizan como variable endógena la tasa de crecimiento medio del Producto Bruto Interno per cápita y como variables explicativas el Producto Bruto Interno per cápita en el año 1965, las tasas de escolaridad (proxys del capital humano), la expectativa de vida, la tasa de fertilidad y dos variables dummies que permiten captar diferencias en el nivel de ingreso. De las tres variables que representan el nivel de la educación, la única significativa y con el signo esperado es la correspondiente a la educación primaria.

Utrera (1999) también analiza el crecimiento de las economías de América Latina y encuentra en una muestra de corte transversal para 20 países que las variables proxys de capital humano (tasas de escolaridad primaria y secundaria), la esperanza de vida al nacer y la inversa de la mortalidad infantil influyen positivamente sobre el crecimiento, mientras que la tasa de escolaridad terciaria, el gasto público en salud y educación y la tasa de analfabetismo impactan negativamente sobre esta variable.

En síntesis, si bien en el ámbito teórico existe consenso sobre el papel que desempeña el capital humano como motor de crecimiento económico, en el aspecto empírico aún quedan algunos aspectos sobre los cuales es necesario seguir investigando. Uno de ellos se refiere a la crítica que generalmente se le hace a los estudios empíricos que utilizan la variable capital humano y es que las variables de educación como las tasas de escolaridad y el promedio de años de educación adquirido son medidas imperfectas del componente educacional del capital humano. Estas miden la cantidad pero no la calidad de la educación lo que podría debilitar la utilidad de las comparaciones internacionales.

\section{I.2.1. Problemas de medición}

En la literatura relativa a la teoría del crecimiento, el capital humano aparece como un 
factor de producción reproducible, parecido en principio al capital físico, tanto en su concepto como en los determinantes del ritmo de acumulación, que no son otra cosa que los determinantes de un proceso de inversión. Esto es, se parte de la hipótesis de que los individuos consideran la educación como una inversión más y deciden llevarla o no a cabo en función de los costes que les supone, y de los presuntos beneficios futuros y de consumo directo que les reporta la escolarización.

En la medición del capital humano es importante considerar si este es considerado como un stock o como un flujo (medido por las tasas de escolaridad). Esta diferencia es importante por dos razones (Psacharopoulos y Arraigada, (1986)). Primero, el rezago en el tiempo entre inversión en educación, medida como tasas de escolaridad y adiciones al stock de capital humano, es mayor que en otro factor de producción. Segundo, la inversión en educación puede ser que nunca redunde en adiciones al stock de capital humano debido a pérdidas en el proceso. Luego, las tasas de escolaridad pueden dar una representación inadecuada cuando se las utiliza para otorgar prioridades relativas en la inversión en educación. Cuando el capital humano es medido como un stock los resultados no son concluyentes, y abundan en la literatura esfuerzos por construir series de datos que reflejen adecuadamente el stock de capital humano de los países.

A pesar de sus limitaciones, las tasas de escolaridad han sido una de las opciones más utilizadas en numerosos estudios, ya que están disponibles para un gran número de países y se encuentran desagregadas en los tres niveles de educación: primario, secundario y terciario. Las tasas de escolaridad bruta relacionan el número total de alumnos para un determinado nivel en relación con el grupo de población al que pertenecen según lo que las leyes determinan para dicho nivel. En cambio, las tasas de escolaridad neta consideran únicamente los alumnos que corresponden según el rango de edad. ${ }^{4}$

Si bien las tasas de escolaridad bruta tienen severas deficiencias como medidas del stock de capital humano y las tasas de escolaridad neta son más apropiadas para la medición del mismo, estas últimas no están disponibles en todos los países en vías de desarrollo. Un problema adicional es el hecho que en los países subdesarrollados los datos sobre estas tasas no son totalmente confiables y los mismos se refieren al número de alumnos que inician el año escolar pero no contempla la deserción durante el período.

En general, ambas tasas tienen deficiencias como medida del stock de capital humano ya que miden los flujos de escolarización mientras que la acumulación de estos flujos genera los futuros stocks de capital humano. Como el proceso de escolarización lleva varios años el rezago entre flujos y stocks es grande.

Otra variable de amplia utilización y de fácil disponibilidad es la de la tasa de analfabetismo adulta. Esta tasa mide un stock de capital humano en la población de 25 años y más mientras que las tasas de escolaridad miden flujos de educación. También tiene cierta dificultad en cuanto a la comparación internacional. La interpretación del término analfabetismo dada por la UNESCO en 1958 varía entre los países y a lo largo del tiempo. Un problema adicional del uso de esta variable es que la alfabetización es sólo el primer paso en el sendero de la formación del capital humano y por lo tanto su utilización implicaría presuponer que la educación más allá de la inicial no contribuye a aumentos significativos en la productividad.

Intentando salvar estas deficiencias, muchos han sido los esfuerzos destinados a la elaboración de bases de datos del stock de capital humano, terreno en el que no existe una metodología única. Uno de los primeros intentos en esta dirección es el trabajo realizado por Psacharopoulos y Arraigada (1986) que han utilizado los datos censales para compilar datos de la composición educacional de la fuerza laboral de personas mayores de 25 años para 99 países. Los niveles de educación cubren cinco categorías: primaria incompleta, primaria completa, secundaria

\footnotetext{
${ }^{4}$ Véase Barro y Lee (1993) para una ampliación de esta distinción.
} 
incompleta, secundaria completa y terciaria.

Más recientemente, ha habido varios intentos de construir series de datos más completos con la educación adquirida que proveen una cobertura temporal más amplia y puedan ser utilizados en las regresiones de crecimiento ya que éstas requieren de la mayor cantidad de datos tanto de países como de años posibles. Kyriacou (1991) estima los años medios de educación de la fuerza laboral a partir de los resultados de Psacharopoulos y Arraigada combinados con los datos de UNESCO y proporciona datos con intervalos de 5 años durante el período 1965-1985 para 111 países. Lau, Jamison y Louat (1991) utilizan un método de inventarios perpetuos y datos anuales de tasas de escolaridad para construir estimaciones de los niveles de educación de la fuerza laboral.

Barro y Lee (1993), con un método más sofisticado, en principio superior, de inventario permanente ${ }^{5}$ y con la información disponible sobre flujos de escolarización y composición por edades de la población, ofrecen el porcentaje de población, por sexos, que ha alcanzado cada nivel de educación. El "set" de datos contiene observaciones para 129 países y cubre el período 19601985 con intervalos de 5 años. Estos datos están actualizados y mejorados en dos trabajos más recientes (Barro y Lee, 1996, 2000), donde las series se extienden hasta el año 1995 e incorporan indicadores de la calidad educativa.

Nehru, Swanson y Dubey (1995) siguen el mismo procedimiento de Lau, Jamison y Louat (1991) y elaboran series con los datos de tasas de escolaridad utilizando el método de inventario permanente ajustado por mortalidad. Realizan las series para una muestra de 85 países industrializados y en desarrollo para el período 1965-87. Introducen notables mejoras al incorporar ajustes por el grado de repetición y de deserción. Este ajuste es importante, sobre todo en países desarrollados donde las cifras pueden estar sobrestimadas hasta en un $25 \%$.

Todos estos estudios pueden ser divididos en dos grupos, de acuerdo a si utilizan los datos censales y las tasas de escolaridad a la vez o emplean sólo estas últimas.

Ambos grupos de autores emplean estimaciones de probabilidades de supervivencia por edades específicas construidas para un país representativo de cada región. La diferencia con la estimación de Barro y Lee es que éstos no realizan estimaciones específicas por edad lo que podría sesgar los resultados si los niveles de educación adquirida difieren sustancialmente entre los diferentes grupos de edad. Es importante destacar que, por otro lado, estos estudios ignoran completamente los datos censales en cuanto a niveles de educación. Para justificar esta decisión, Nehru et al. observan que las publicaciones censales generalmente no indican los años actuales de escolaridad de cada individuo (solo indican si han completado cierto nivel de educación y/o lo han comenzado) y generalmente proveen información solo para la población superior a 25 años de edad. Esto provocaría cierta arbitrariedad en las estimaciones de promedios de años de escolaridad al omitir los segmentos jóvenes de población sobre todo en los países menos desarrollados donde este grupo de edad es típicamente mayor y más educado que cohortes mayores.

Estas diferencias metodológicas entre los distintos estudios serían de relativa importancia si todas reflejaran un panorama semejante en cuanto a los niveles de educación alcanzados por los países y su evolución a lo largo del tiempo. Sin embargo, este no es el caso, ya que diferentes fuentes muestran variaciones significativas en términos de la posición relativa de los países cuando se analiza la información desagregada (y no por grandes grupos como por ejemplo países más y menos desarrollados).

Se puede concluir que, si bien el capital humano es multifacético e incluye un completo "set" de atributos humanos y el stock de capital adquirido por los individuos es difícil de cuantificar con precisión, en los últimos años existe consenso entre los autores en utilizar una

\footnotetext{
${ }^{5}$ Cada país tiene una base de medición censal estimada al menos para un año que provee una base para los flujos de escolaridad.
} 
medida de educación que refleje el stock de capital humano incorporado por los individuos, como podría ser el promedio de años de educación adquirido. Temple (2000), Barro (2000), De la Fuente y Domenech (2000), entre otros, consideran que esta es una variable más interesante desde el punto de vista teórico para medir el stock existente de capital humano de un país cuando se analiza la información en forma agregada, ya que al ser las tasas de escolaridad una variable flujo no representan con fidelidad los cambios el stock de capital humano, principalmente en períodos de transición educacional. Por otra parte, la simultaneidad resultante de la posibilidad de que estos flujos de inversión en capital humano puedan ser provocados por altas tasas de crecimiento confunde su interpretación en los modelos de regresión "cross-country".

\section{I.3. Capital humano: cantidad y calidad}

Para mantener la base de capital humano que requiere el crecimiento económico es preciso transformar con profundidad la prestación de los servicios de educación, ya que la inversión en capital humano por sí sola no puede garantizar un crecimiento más rápido. Puede ocurrir que ese capital humano no se utilice en forma apropiada, o que esa inversión en capital humano resulte desacertada o de calidad mediocre con lo cual muchas veces, los gastos en recursos humanos no permiten obtener la cantidad, la calidad, o el tipo de capital humano que habría sido posible si los recursos se hubieran gastado en forma más eficiente. Por lo tanto, se deben tener en cuenta las prioridades en materia educativa concentrándose principalmente en la calidad en la prestación de los servicios.

La calidad ha llegado a ser una de las preocupaciones fundamentales de la educación, ello obedece a que la satisfacción de las expectativas y las necesidades de la sociedad con relación a la educación depende de la calidad del personal, sus programas, sus estudiantes así como de su infraestructura y su entorno académico. Sin embargo, no es fácil el criterio de selección de los indicadores más apropiados para determinar esta calidad. Conceptualmente, el logro de la calidad educativa puede ser medida por varios indicadores que permiten suministrar información de tipo cuantitativo y cualitativo. Los indicadores se pueden clasificar atendiendo a múltiples criterios (Calero Martínez (1999)). Existen indicadores de insumo que tienen que ver con los recursos humanos y financieros empleados por las instituciones de educación. Ejemplo de esto es la proporción de presupuesto público destinado al financiamiento de la educación y porcentaje de recursos propios en relación con el total de recursos. Por otro lado están los indicadores de proceso, de producto y de resultado. Los primeros se refieren a los medios a través de los cuales los insumos se transforman en producto. Estos indicadores se refieren al número de alumnos por aula, razón profesor alumno, proporción de profesores titulares respecto de los auxiliares y costo por alumno. Los indicadores de producto incluyen desde los efectos como el nivel de aprendizaje alcanzado por el alumno hasta cuestiones relacionadas con la igualdad. Estos indicadores se refieren a la proporción de egresados sobre ingresantes, a la proporción de egresados pertenecientes a familias de menores ingresos. Finalmente, los indicadores de resultado hacen referencia a la interacción entre los productos educativos y el entorno social tales como proporción de egresados que se encuentran desempleados, distribución de la población según nivel educativo y niveles de ingreso.

La motivación para incorporar indicadores de calidad en el análisis de las diferencias en la cantidad de la educación es simple. Las variaciones en la calidad educativa implican que un año de escolaridad obtenido en un momento diferente, en un país diferente, o en una escuela diferente puede no ser equivalente a otro año en circunstancias distintas. Los sistemas educativos varían ampliamente entre los países en términos de organización, recursos y preparación de docentes y alumnos que llegan a la escuela. Los gastos en educación por alumno varían ampliamente entre los países, las condiciones educativas cambian desde aulas a la intemperie con docentes de poca preparación hasta modernas aulas equipadas con tecnología virtual y docentes con niveles de posgrado. El año promedio escolar varía desde 100 hasta 200 días de clase por año. Todas estas diferencias tienen fuertes implicancias al considerar solamente como proxy del capital humano una variable que tome en cuenta los años promedio de escolaridad adquiridos. 
Hanushek y Kim (1995) proponen medir la educación adquirida utilizando las calificaciones en tests internacionales de habilidades cognitivas en matemáticas y ciencia. Sus resultados indican que estos tests están positivamente relacionados con el crecimiento del PBN per cápita en las regresiones cross-country. Este resultado indica que la calidad de la educación, en adición a la cantidad, es un ingrediente importante en el análisis de la relación capital humano y crecimiento económico.

Barro (1998) también utiliza los resultados de los tests internacionales para incorporar la calidad educativa en las regresiones de crecimiento. Concluye que, dada la calidad de la educación representada por los resultados en los tests internacionales, la cantidad de educación adquirida, medida por los años promedio de educación alcanzados por la población adulta masculina en el nivel secundario y terciario, es todavía positiva y significativa con relación al crecimiento posterior.

Un tema importante dentro de la discusión sobre la calidad educativa ha sido la relación entre las medidas de calidad de resultado (ingresos futuros, rendimientos en exámenes y otros) y los recursos utilizados en las escuelas. Para Hanushek y Luque (2001) debido al hecho de que las medidas de calidad directa no están disponibles, surge la pregunta de si la medición simple de los recursos empleados en las escuelas puede ser un buen sustituto en la medición de la calidad educativa. Conceptualmente, el logro estudiantil puede ser influenciado por los recursos disponibles para los estudiantes en las escuelas. Estos recursos pueden ser medidos por varios indicadores tales como razón alumno-docente, gastos por alumno, salario y nivel de educación docente, duración del año escolar disponibilidad de material en el aula, etc.. En general aún subsiste cierta controversia sobre si los recursos escolares influyen positivamente en el rendimiento estudiantil y los estudios empíricos encuentran solo en algunos casos evidencia positiva. ${ }^{6} \mathrm{Al}$ respecto, Heyneman y Loxley (1983) encuentran que las diferencias en los recursos educativos tienen más relación con los rendimientos estudiantiles en los países en vías de desarrollo que en Estados Unidos. ${ }^{7}$ Card y Krueger (1992) destacan una correlación positiva entre calidad educativa (medida por los indicadores mencionados) y logro estudiantil, medido por rendimientos futuros. Barro y Lee (1998) realizan un estudio empírico para analizar los determinantes de la calidad educativa con un nuevo set de datos para una amplia muestra de países. Los resultados muestran que los conocimientos de la familia así como los recursos disponibles en las escuelas están positivamente relacionadas con el rendimiento educativo medido por los resultados de tests internacionales. Harbison y Hanusheck (1992) también encuentran evidencia de la importancia de la educación de los docentes y de los recursos en el aula para el logro estudiantil de los alumnos en Brasil.

En conclusión, la evidencia tanto de una gran variedad en la calidad de la educación, así como de su importante impacto en los logros estudiantiles, explica el renovado interés por las políticas educativas dentro de la literatura teórica.

\section{UN MODELO DE CRECIMIENTO CON CAPITAL HUMANO Y CALIDAD}

En esta sección se analiza la influencia del capital humano sobre el crecimiento económico incorporando la calidad educativa como una variable de relevancia dentro de esta relación. Siguiendo el enfoque de la versión extendida del modelo neoclásico, se estimarán diferentes regresiones que incluyen el capital humano como un factor más en la función de producción, medido este a través del nivel educativo de la población activa.

\footnotetext{
${ }^{6}$ Distintas opiniones sobre el tema pueden consultarse en Hedges, Laine y Greenwald (1994) y Hanushek (1996).

${ }^{7}$ Una discusión más amplia de los resultados de estimaciones sobre los efectos de los recursos se puede encontrar en Hanusheck $(1986,1997)$
} 


\section{II.1. Datos y variables utilizadas}

Se realiza un análisis de panel de datos y otro de cross section para una muestra de 33 países en el período 1960-2000. En ambos casos, la variable dependiente es la tasa de crecimiento del PBI per cápita. A su vez, como variables de control, siguiendo los resultados de Levine y Renelt (1992) se utiliza un "set" de variables que estos autores encontraron robustas para explicar el crecimiento del PBI per cápita y se investiga principalmente la magnitud y estabilidad de la influencia del capital humano y su calidad. Estas variables son: el PBI inicial ${ }^{8}$, la cantidad de capital humano que se representa a través del promedio de años de educación adquiridos en el sector secundario por la población adulta mayor de 25 años al inicio de cada período ${ }^{9}$, el coeficiente de inversión sobre el producto y la tasa de crecimiento de la población. Se utilizan también como variables explicativas aquéllas que indican la calidad de la educación. Estas variables son de dos clases. Por un lado, el gasto por alumno como porcentaje del PBI, la razón alumno-docente y los días de duración del año escolar (solo para el análisis cross-section), que son medidas convencionales de los recursos escolares que pueden influir en la calidad educativa. Por otra parte, se utiliza también una variable de elaboración propia que incorpora los resultados obtenidos en tests cognitivos internacionales y como sugieren Hanushek y Kim (1995), captura las diferencias en las habilidades individuales de los agentes económicos y da un panorama más completo de la calidad del capital humano. La información de los resultados de los tests internacionales se encuentra disponible para las áreas de ciencias, matemáticas y lectura. Una deficiencia que tienen estos datos es que corresponden a diferentes años y están más completos para la década del '90. Los datos disponibles se utilizaron para construir una medida basada en los resultados de los tests de ciencias ${ }^{10}$, y completada con los resultados en lectura. Esta tarea se realizó utilizando la relación promedio entre los resultados de ciencias y lectura para los países que tenían disponibles ambos resultados.

El resto de los datos utilizados en este trabajo fueron obtenidos de las series del World Bank y de Barro-Lee (2000)*. Siguiendo un criterio de selección basado en la disponibilidad de datos, la muestra incluye 33 países en la que la principal limitación fueron los datos referidos a educación. En el caso del panel de datos se utilizan nueve períodos de cinco años cada uno desde 1960 a 2000, mientras que en la cross-section se utilizan los promedios de las variables.

\section{II.2. La relación “capital humano - crecimiento económico”}

A continuación se presenta el análisis de las regresiones realizadas para analizar el impacto de las variables de capital humano y de calidad educativa sobre el crecimiento económico de los países de la muestra ordenados por su nivel de ingreso siguiendo la clasificación del World Bank. Se utiliza primeramente la técnica de panel de datos ya que las regresiones cross-section utilizan variables medidas como el valor promedio a lo largo del tiempo para cada país, con lo cual se pierde la información proveniente de la evolución temporal de las variables y en este caso es difícil controlar la heterogeneidad entre países. Las diferencias culturales, institucionales, tecnológicas

\footnotetext{
${ }^{8}$ Empíricamente se incorpora el nivel inicial del producto per cápita en la ecuación de crecimiento en forma logarítmica. El coeficiente estimado de esta variable representará entonces la tasa de convergencia.

${ }^{9}$ La elección de esta variable como proxy del stock capital humano sigue a otros estudios empíricos anteriores que la consideran de gran poder explicativo para el crecimiento económico ( Barro (1998, 2000), Hanushek y Kim (1995)).

${ }^{10}$ Barro (2000) encuentra que esta variable es altamente significativa para predecir el crecimiento económico.

* CRECPBI: promedio de la tasa de crecimiento del PBI per cápita en dólares a valores constantes a precios de mercado. (Elaboración propia en base a datos de (WB)).Log GDP: Ingreso per cápita inicial,1960( WB). POP: Tasa de Crecimiento de la población (WB). INV: Razón entre la inversión y el PBI (WB). GASPRI: Promedio de la razón del gasto en educación primaria y el PBI (BL).GASEC: Promedio de la razón del gasto en educación secundaria y el PBI (BL). SYR: Promedio de los años de educación adquiridos en el sector secundario por la población adulta mayor de 25 años al inicio de cada período (BL). LSC: Porcentaje de población activa que ha alcanzado un nivel de educación secundaria para la población mayor de 25 años (BL). RAZPRI: Promedio de la razón alumno- docente en el sector terciario (BL). RAZSEC: Promedio de la razón alumno- docente en el sector secundario (BL).TEST: medida de calidad educativa que incorpora los resultados de los tests cognitivos internacionales. (Elaboración propia sobre la base de (BL)).
} 
existentes entre los países no son captadas por una regresión simple cross- section, con lo cual la incidencia de estos factores queda en el residuo de la regresión. Si estos factores están correlacionados positivamente con las variables incluidas en el modelo la estimación de los parámetros estaría sesgada.

El panel de datos se ha formulado como un modelo de efectos fijos. Se supone que las diferencias entre países pueden ser captadas mediante un parámetro desconocido estimado en el término constante. Para contrastar la hipótesis que los términos constantes son todos iguales se realizó el test de Wald que indica si existen particularidades en los países que sugieran que es necesario tenerlas en consideración o, en su defecto, estimar una ecuación para cada uno de los mismos. A priori se podría pensar que dichos efectos existen, es decir, el marco institucional que presenta Argentina no tiene porqué ser igual al peruano, o, las características de un país pequeño como Trinidad Tobago pueden diferir de las de uno grande como Estados Unidos. De esta manera, el valor del test obtenido confirma la existencia de los efectos fijos, puesto que se rechaza la hipótesis nula de ausencia de los mismos, con un nivel de significatividad del $1 \%$.

Luego del análisis de panel de datos y para comparar con los resultados del mismo también se realizará un análisis de cross-section.

En la tabla 1 se presentan los resultados econométricos de las regresiones realizadas. En primer lugar se analiza para la muestra total y luego por grupo de países de acuerdo a los distintos niveles de ingreso. En todas las estimaciones se toman en cuenta las variables encontradas por Levine y Renelt (1992) como robustas para explicar el crecimiento económico: el PBI inicial (Log GDP), el capital humano inicial (SYR), el coeficiente de inversión sobre el producto (INV) y la tasa de crecimiento de la población (POP). Luego se incorporan en las estimaciones las variables de calidad de la educación (GASPRI, GASEC, RAZPRI y RAZSEC) que indican la disponibilidad de recursos educativos. Finalmente se reemplazan estas variables por una variable de calidad que contempla los resultados de los tests cognitivos (TEST).

Las primeras tres estimaciones corresponden a la muestra total de países. En la columna 2 se encuentran los signos esperados. La variable logGDP es significativa11. El ingreso inicial afecta en forma negativa y significativa al crecimiento económico, resultado compatible con la hipótesis de convergencia condicional. La tasa de inversión es significativa y con el signo esperado. Es razonable pensar que esta variable determina, pero a la vez está determinada por el crecimiento económico por lo que podría haber alguna controversia sobre la causalidad de la misma. Sin embargo, el principal interés de este trabajo es ver la sensibilidad de las estimaciones de capital humano con la inclusión de estas variables. La variable de capital humano SYR tiene el signo esperado y es significativa. En cuanto al crecimiento poblacional las estimaciones indican una relación negativa y significativa entre tasa de crecimiento del PBI per cápita y crecimiento de la población. Este resultado coincide con los hallados en la literatura empírica e indica que un mayor ingreso lleva a los países a una disminución en su tasa de natalidad. En la columna 2 se presentan los resultados de las regresiones al incorporar las variables explicativas de la calidad del capital humano: gasto en educación (GASPRI y GASEC) y razón alumno docente (RAZPRI Y RAZSEC) que expresan los recursos educativos disponibles en el aula. Con la incorporación de estas variables se encuentra que las variables de control se mantienen con los niveles de significatividad y los signos de la regresión anterior. Dentro de las variables de calidad la única que resulta significativa es el gasto por alumno en educación primaria (GASPRI) ${ }^{12}$, aunque el signo no es el esperado ya que indica que un mayor gasto por alumno en educación primaria es acompañado de un menor crecimiento del PBI. El resto de las variables de calidad (GASEC, RAZPRI Y RAZSEC) no resultaron ser significativas por lo tanto no explicarían para los países que se están analizando el crecimiento económico. En la columna tres se incorpora la variable TEST elaborada en base a los

\footnotetext{
${ }^{11}$ Entendiéndose como nivel crítico el 5\% (es decir un p-value menor que 0.05) para considerar a una variable significativa.

${ }^{12}$ Este resultado es coincidente al enconrtrado por Hanushek y Kim (1995).
} 
resultados de los test cognitivos a nivel internacional con datos de Barro y Lee (2000). Aquí se encuentra que tanto la medida de cantidad de capital humano SYR como la de calidad TEST son significativas. El resto de las variables permanecen con los signos y niveles de significatividad de la regresión anterior. En este caso hay una notable mejoría en el $\mathrm{R}_{2}$ que alcanza un valor de 0.51 .

Cuando las estimaciones se realizan por niveles de ingreso, en forma compatible con la hipótesis del trabajo, se encuentra que los resultados cambian según se trate de países con nivel de ingreso alto, medio alto o bajo y medio bajo ${ }^{13}$. Las variables PBI inicial y tasa de inversión se mantienen signicativas y con el signo esperado para todos los niveles de ingreso. Con respecto al crecimiento de la población en el grupo de países con nivel de ingreso alto se encuentra una relación negativa y significativa entre tasa de crecimiento del PBI y tasa de crecimiento de la población al igual que en la muestra total de países. En los otros dos grupos de países se encuentra que esta relación es positiva y significativa ${ }^{14}$. La variable proxy del capital humano (SYR) contribuye positivamente a explicar las variaciones en la tasa de crecimiento del PBI per cápita en todos los grupos analizados. Al incorporar las variables explicativas de la calidad del capital humano se advierte que los resultados encontrados coinciden con los de la regresión correspondientes a la muestra total de países: la única que resulta significativa es el gasto por alumno en educación primaria (GASPRI) para el grupo de países con niveles de ingreso alto y bajo y medio bajo aunque el signo es el esperado solo en este último caso. Es decir que esta variable explicaría el crecimiento económico en los países con niveles de ingreso bajo y medio bajo. El resto de las variables de calidad (GASEC, RAZPRI Y RAZSEC) no resultaron ser significativas por lo tanto no explicarían el crecimiento económico para ninguno de los grupos de países analizados. Cuando se incorpora la variable TEST, elaborada en base a los resultados de los test cognitivos a nivel internacional se encuentra que no varían significativamente los signos y niveles de significatividad de las variables de control. Sólo en los países de ingreso bajo y medio bajo la variable de cantidad de capital humano deja de ser significativa. Esto podría comprenderse debido a que en los países con este nivel de ingreso importa más la calidad de la educación que la cantidad de la misma. La variable (TEST) es significativa en los países con niveles de ingreso medio alto y bajo y medio bajo pero no en los de ingreso alto. Esto podría tener su explicación debido a que los países con nivel de ingreso alto han alcanzado un nivel de calidad tal que un aumento en la misma no alcanza a modificar el crecimiento del PBI per cápita observado, lo cual se podría asociar a la existencia de rendimientos decrecientes del capital humano. Cabe destacar que en todos los grupos de países se encuentra una notable mejoría del $\mathrm{R}^{2}$ en las regresiones con la inclusión de esta variable.

De los resultados de las regresiones anteriores se puede concluir que una medida de calidad que refleje los resultados de tests cognitivos en el orden internacional resulta ser superior que aquellas medidas que indican los recursos disponibles en el ámbito escolar cuando se analiza el total de la muestra de países. Este resultado coincide con el encontrado por Hanushek y Kim (1995). Sin embargo, cuando el análisis se realiza por niveles de ingreso la variable de calidad que refleja los resultados de los tests cognitivos no resulta ser significativa en los países con ingreso alto reafirmando la hipótesis planteada en el trabajo de que la calidad del capital humano como factor explicativo del crecimiento económico varía en países con diferentes niveles de ingreso per cápita.

\footnotetext{
${ }^{13}$ Estos dos grupos fueron analizados en forma conjunta debido a la poca disponibilidad de los datos.

${ }^{14}$ Existe una amplia literatura (Ray,D.(1998), Bardhham,P.(1995)) que intenta explicar los motivos de las mayores tasas de fertilidad en países con menores niveles de ingreso debido a múltiples factores como falta de control de la natalidad, desempleo, promiscuidad, etc.
} 
Cuadro 1 - Regresiones de crecimiento por nivel de ingreso de países

\begin{tabular}{|c|c|c|c|c|c|c|c|c|c|c|c|c|}
\hline & \multicolumn{3}{|c|}{ Muestra total } & \multicolumn{3}{|c|}{ Nivel de ingreso alto } & \multicolumn{3}{|c|}{ Nivel de ingreso medio alto } & \multicolumn{3}{|c|}{$\begin{array}{c}\text { Nivel de ingreso bajo y medio } \\
\text { bajo }\end{array}$} \\
\hline & Reg.1 & Reg.2 & Reg.3 & Reg.1 & Reg.2 & Reg.3 & Reg.1 & Reg.2 & Reg.3 & Reg.1 & Reg.2 & Reg.3 \\
\hline Log GDP & $\begin{array}{c}-0.95 \\
(0.003)\end{array}$ & $\begin{array}{c}-0.95 \\
(0.003)\end{array}$ & $\begin{array}{c}-1.3 \\
(0.006)\end{array}$ & $\begin{array}{c}-.49 \\
(0.000)\end{array}$ & $\begin{array}{c}-0.36 \\
(0.000) \\
\end{array}$ & $\begin{array}{c}-0.42 \\
(0.041)\end{array}$ & $\begin{array}{l}-0.83 \\
(0.04)\end{array}$ & $\begin{array}{c}-0.54 \\
(0.007)\end{array}$ & $\begin{array}{c}-1.46 \\
(0.013) \\
\end{array}$ & $\begin{array}{c}-0.8 \\
(0.022)\end{array}$ & $\begin{array}{c}-0.32 \\
(0.034) \\
\end{array}$ & $\begin{array}{c}-0.61 \\
(0.032)\end{array}$ \\
\hline INV & $\begin{array}{c}0.2 \\
(0.001) \\
\end{array}$ & $\begin{array}{c}0.22 \\
(0.001) \\
\end{array}$ & $\begin{array}{c}0.3 \\
(0.006) \\
\end{array}$ & $\begin{array}{c}0.99 \\
(0.007) \\
\end{array}$ & $\begin{array}{c}0.14 \\
(0.01) \\
\end{array}$ & $\begin{array}{c}0.39 \\
(0.013) \\
\end{array}$ & $\begin{array}{c}0.28 \\
(0.001) \\
\end{array}$ & $\begin{array}{c}0.3 \\
(0.000)\end{array}$ & $\begin{array}{c}0.43 \\
(0.008) \\
\end{array}$ & $\begin{array}{c}0.12 \\
(0.04) \\
\end{array}$ & $\begin{array}{c}0.07 \\
(0.04)\end{array}$ & $\begin{array}{c}0.13 \\
(0.03) \\
\end{array}$ \\
\hline SYR & $\begin{array}{c}0.92 \\
(0.01) \\
\end{array}$ & $\begin{array}{c}1.07 \\
(0.004) \\
\end{array}$ & $\begin{array}{c}0.9 \\
(0.019) \\
\end{array}$ & $\begin{array}{c}0.65 \\
(0.039) \\
\end{array}$ & $\begin{array}{c}0.81 \\
(0.02) \\
\end{array}$ & $\begin{array}{c}0.12 \\
(0.008) \\
\end{array}$ & $\begin{array}{c}1.17 \\
(0.021) \\
\end{array}$ & $\begin{array}{c}1.83 \\
(0.05) \\
\end{array}$ & $\begin{array}{c}6.67 \\
(0.03) \\
\end{array}$ & $\begin{array}{c}1.18 \\
(0.04) \\
\end{array}$ & $\begin{array}{c}1.57 \\
(0.03) \\
\end{array}$ & $\begin{array}{c}1.01 \\
(0.33) \\
\end{array}$ \\
\hline POP & $\begin{array}{c}-0.63 \\
(0.002)\end{array}$ & $\begin{array}{c}-0.62 \\
(0.002)\end{array}$ & $\begin{array}{c}-1.39 \\
(0.003)\end{array}$ & $\begin{array}{l}-1.45 \\
(0.002)\end{array}$ & $\begin{array}{c}-1.53 \\
(0.001)\end{array}$ & $\begin{array}{c}-0.91 \\
(0.002)\end{array}$ & $\begin{array}{c}0.81 \\
(0.057)\end{array}$ & $\begin{array}{c}2.45 \\
(0.015)\end{array}$ & $\begin{array}{c}8.44 \\
(0.01)\end{array}$ & $\begin{array}{c}0.71 \\
(0.001)\end{array}$ & $\begin{array}{c}0.27 \\
(0.003)\end{array}$ & $\begin{array}{c}0.41 \\
(0.002)\end{array}$ \\
\hline GASPRIM & & $\begin{array}{l}-0.009 \\
(0.03) \\
\end{array}$ & & & $\begin{array}{l}-0.08 \\
(0.03) \\
\end{array}$ & & & $\begin{array}{l}-0.12 \\
(0.39)\end{array}$ & & & $\begin{array}{c}0.45 \\
(0.05)\end{array}$ & \\
\hline GASEC & & $\begin{array}{l}-0.00 \\
(0.94) \\
\end{array}$ & & & $\begin{array}{l}-0.03 \\
(0.41) \\
\end{array}$ & & & $\begin{array}{c}-0.00 \\
(0.82) \\
\end{array}$ & & & $\begin{array}{l}-0.01 \\
(0.44) \\
\end{array}$ & \\
\hline RAZPRIM & & $\begin{array}{l}-0.01 \\
(0.64)\end{array}$ & & & $\begin{array}{l}-0.03 \\
(0.62)\end{array}$ & & & $\begin{array}{l}-0.18 \\
(0.09)\end{array}$ & & & $\begin{array}{l}0.06 \\
(0.5)\end{array}$ & \\
\hline RAZSEC & & $\begin{array}{l}0.01 \\
(0.8) \\
\end{array}$ & & & $\begin{array}{c}0.02 \\
(0.64) \\
\end{array}$ & & & $\begin{array}{l}0.11 \\
(0.4) \\
\end{array}$ & & & $\begin{array}{l}-0.24 \\
(0.13) \\
\end{array}$ & \\
\hline TEST & & & $\begin{array}{c}0.13 \\
(0.002) \\
\end{array}$ & & & $\begin{array}{c}0.93 \\
(0.32) \\
\end{array}$ & & & $\begin{array}{c}0.37 \\
(0.002) \\
\end{array}$ & & & $\begin{array}{c}0.37 \\
(0.005) \\
\end{array}$ \\
\hline $\begin{array}{c}\mathrm{R}^{2} \\
\text { Ajustado }\end{array}$ & 0.26 & 0.29 & 0.51 & 0.39 & 0.45 & 0.55 & 0.33 & 0.42 & 0.74 & 0.2 & 0.21 & 071 \\
\hline D-W & 2.30 & 2.34 & 4.20 & 2.19 & 2.21 & 6.4 & 2.16 & 2.6 & 3.82 & 2.6 & 2.48 & 3.41 \\
\hline
\end{tabular}

Los números entre paréntesis representan las p-value asociadas al valor del estádistico "t“"

i.Países con nivel de Ingreso Bajo: PBI per cápita en el año 1998 de u\$s 760 o menos. Malawi- Zimbawe.

ii.Países con nivel de Ingreso Medio Bajo: PBI per cápita en el año 1998 entre u\$s 760 y u\$s 3030. Colombia-Filipinas-Swazilandia-Tailandia.

iii.Países con nivel de ingreso Medio Alto: PBI per cápita en el año 1998 entre u\$s 3031 y u\$s 9360. Brasil-Botswana-Chile-Hungría-Korea-Trinidad y Tobago-Venezuela.

iv.Países con nivel de Ingreso Alto: PBI per cápita en el año 1998 superior a u\$s 9361. Austria-Bélgica-Canadá-Estados Unidos-España-Finlandia-Grecia-HongKong-Israel-IrlandaItalia-Japón-Holanda-Noruega-Nueva Zelanda-Portugal-Suecia-Singapur-ReinoUnido. 
Para corroborar los resultados del análisis de panel de datos se realizó un análisis de corte transversal para verificar el impacto de las variables de capital humano y de calidad educativa sobre el crecimiento económico de los países de la muestra clasificados por su nivel de ingreso. Se realizaron cuatro conjuntos de tres regresiones, tomando en todos los casos las cuatro variables consideradas robustas por Levine y Renelt. En las primeras no se incluyen las medidas de calidad, en las segundas se incluyen las variables de calidad que indican la disponibilidad de recursos educativos, luego se reemplazan estas medidas por la variable TEST que contempla los resultados de los tests cognitivos y por último se utilizan variables dummy para captar las diferencias de acuerdo a los niveles de ingreso de los países. Los resultados arrojados con estas regresiones confirman los obtenidos con la técnica de panel de datos permitiendo concluir que tanto con el método de panel de datos como con el de cross section, se puede concluir que las medidas de calidad que reflejan los resultados de tests cognitivos en el orden internacional son superiores que aquellas medidas que indican los recursos disponibles en el ámbito escolar para explicar el crecimiento de un país. Este resultado es coincidente con el encontrado por Hanushek y Kim (1995) y Barro (1998, 2000).

\section{CONCLUSIONES}

Los modelos de capital humano demuestran la forma en que la educación posibilita que todo el proceso de producción se beneficie con las externalidades que una sociedad más educada genera. La mano de obra más capacitada utiliza el capital de manera más eficiente, con lo cual pasa a ser más productiva. De esta manera, la elevación del nivel de educación provoca un aumento de la eficiencia de todos los factores de producción. La cuestión fundamental es el alcance de la inversión en capital humano para contribuir al crecimiento económico.

La calidad educativa es un componente importante del capital humano cuando se compara una muestra de países en el ámbito internacional. Las variables que se utilizan como proxys del capital humano en los estudios empíricos son medidas imperfectas del componente educacional del capital humano, ya que miden la cantidad pero no la calidad de la educación y por lo tanto no toman en cuenta limitaciones de estructura y funcionamiento del sistema educativo que obstaculizan un mejor aprovechamiento del capital humano.

En el presente trabajo se ha comenzado con una revisión de la literatura teórica y empírica de la relación entre capital humano y crecimiento económico. Luego se ha profundizado la investigación de la importancia de la calidad educativa en la formación del capital humano y su influencia en el crecimiento económico a través de un estudio empírico de panel de datos y de cross-section para una muestra de 33 países, agrupados por niveles de ingreso. Para medir la calidad de la educación se utilizaron dos clases de variables. Por un lado, el gasto por alumno como porcentaje del PBI en el ámbito primario y secundario y la razón alumno -docente en los mismos niveles que son medidas convencionales de los recursos escolares que pueden influir en la calidad educativa. Por otra parte, se utilizó una variable que incorpora los resultados obtenidos en tests internacionales que captura diferencias en las habilidades individuales de los agentes económicos y da un panorama más completo de la calidad del capital humano.

De los resultados de las regresiones realizadas tanto de corte transversal como de panel de datos se puede concluir que la calidad de la educación tiene un importante efecto sobre el crecimiento económico de los países a escala global. La inclusión de la variable de calidad educativa TEST mejora notablemente los alcances de las regresiones con capital humano. Los resultados obtenidos en el trabajo son sensibles a la muestra analizada: cuando el análisis se realiza diferenciando a los países de acuerdo con sus niveles de ingreso se encuentra que para los países de ingreso alto la variable de calidad de capital humano no mejora la perfomance de crecimiento de la misma manera que en los países con ingresos inferiores. 
En síntesis, los resultados de este trabajo apoyan la hipótesis que una mayor calidad en la educación tiene un efecto positivo en el crecimiento económico, aportando evidencia empírica que apoya las políticas orientadas a aumentar el stock de capital humano y su calidad en la economía. Este resultado se obtuvo independientemente del método de estimación utilizado. El análisis también sugiere que sería necesario considerar las diferencias en los niveles de ingreso de los países para determinar la importancia de la calidad educativa en el crecimiento económico. Por ejemplo, en función de los resultados obtenidos en el trabajo, una política destinada a aumentar la calidad educativa (medida por los resultados cognitivos de los tests internacionales) no tendría el efecto esperado sobre la tasa de crecimiento en los países de ingreso alto, pero sí lo tendría si el país no perteneciera a este grupo. Es decir que tanto la cantidad como la calidad del capital humano son variables fundamentales a tener en cuenta en el momento de realizar acciones de política económica tendientes a favorecer el crecimiento económico, teniendo en cuenta que no todas las medidas de calidad se comportan de la misma manera.

Una próxima tarea de investigación sería continuar con la línea de Hanushek y Kim para elaborar una variable representativa de la calidad educativa que incorpore los resultados de los tests internacionales. Se trataría de ampliar las observaciones y realizar el análisis para más países lo que permitiría reafirmar las conclusiones de este trabajo. También sería de interés realizar el análisis utilizando algún método de estimación alternativo al de efectos fijos como el System GMM $^{15}$ que permitiría solucionar el problema de la posible endogeneidad entre las variables así como también el de efectos individuales de cada país.

\footnotetext{
${ }^{15}$ El método System GMM fue desarrollado por Arellano y Bover (1995) y Blundell y Bond (1998) como una mejora al Método General de Momentos (GMM). El System GMM estima un sistema de ecuaciones tanto en primeras diferencias como en niveles donde los instrumentos para las series en niveles son los valores rezagados de las series en primeras diferencias.
} 


\section{BIBLIOGRAFIA}

Arrow, K.J.(1962): “The Economic Implications of learning by doing”, Review of Economic Studies, 29, Jun, pp.155-173.

Azariadis, C. y Drazen, A. (1990): "Threshold Externalities in Economic Development". Quarterly Journal of Economics, 90, pp 501-526.

Barro, R. (1990): "Government Spending in a Single model of Endogenous Growth”, Journal of Political Economy, 98,5, pp.103-125. (1991): " Economic Growth in a cross section of Countries", Quarterly Journal of Economics, Vol. CVI, May. ,(1995): Determinants of Economic Growth: a Cross-Country Empirical Study, Massachusets, MIT Press.

,(1998): Human Capital and Growth in Cross-Country Regressions, Harvard University Press. ,(2000): Education and Economic Growth, Harvard University Press.

Barro, R. and Jong-Wha Lee (1993): “International comparisons of Educational Attainment”, Journal of Monetary Economics, 32, pp.363-394. ,(1998): “Schooling Quality in a cross section of Countries”, Development Discussion Paper, $\mathrm{N}^{\circ} 659$, Harvard College.

(2000): International comparisons of Educational Attainment. Updates and Implications, Harvard University.

Barro, R and Sala-i-Martin X.(1991): “Convergence across States and Regions”, Brookings Papers on Economic Activity, 1, pp.107-179. ,(1992) , “Convergence”, Journal of Political Economy 100,(2), April.

Becker, G.(1964): ”Human Capital”, Chicago IL: University of Chicago Press. (Traducción Castellana, Alianza Universidad textos, Madrid,1985).

Benhabib, J. y Spiegel, M (1994): "The role of Human capital in Economic Development. Evidence from aggregate cross-country data”, Journal of Monetary Economics, 34, pp.143-173.

Calero, J. y Martínez Bonal, X., (1999): Política educativa y gasto público en educación. Ed.Pomares-Corredor, Barcelona.

Card, D. y Krueger, A.(1992): "Does school quality matter? Returns to Education and the characteristics of public schools in the United States", Journal of Political Economy, 100.1 .

De la Fuente and R. Domenech (2000): “ Human Capital in Growth Regressions: How much difference does data quality make? OECD Economics Department Working Papers, No. 262.

De la Fuente, A. y Da Rocha J. (1996): " Capital humano y crecimiento: Un panorama de la evidencia empírica y algunos resultados para la OCDE", Moneda y crédito, $\mathrm{N}^{\circ} 203$, pp.43-84.

Elías, S. y Fernández, M. (1999): ”Determinantes del Crecimiento Económico: Un estudio empírico para Latinoamérica", Anales de la XXXIV Reunión Anual de la Asociación Argentina de Economía Política, Rosario, Argentina.

Gemmel, N. (1996): "Evaluating the impacts of human capital stocks and accumulation on Economic Growth: some new evidence”, Oxford Bulletin of Economics and Statistics, 58(I), pp.9-28.

Hanushek, E. y Kim, D.(1995): “Schooling, Labor Force quality, and Economic Growth”, NBER Working Paper $N$ 95399.

Heyneman, S. y Loxley, W.(1983): “ The effect of primary school quality on academic achievement across twenty nine high and low income countries.” American Journal of Sociology, 88, May.

Klenow, P. y Rodriguez-Clare A.(1997): "The Neoclassical Revival in Growth Economics: Has it gone too far?”, NBER Macroeconomics Annual 1997, Volume 12, pp.73-103.

Laing D., Palivos,T. and Wang P.(1995) : "Learning, Matching and Growth", Review of Economic Studies, 62, pp.115-129. 
Laitner, J.(1993): “ Long-run Growth and Human Capital”, Canadian Journal of Economics, XXVI, Nov, pp.796-814.

Lau,L., Jamison, D. and Louat,F.(1991) : "Education and productivity in developing countries: an aggregate production function approach, Report $\mathrm{N}^{\circ} 612$, WPS.

Levine,R. and Renelt D.(1992):” A sensivity analysis of cross-country Growth Regressions.” American Economic Review, 82, No4, pp.942-63.

Mankiw, N.G., Romer, D. y Weil, D.N.(1992): "A Contribution to the empirics of Economic Growth”, Quarterly Journal of Economics, (107), 3, pp.407- 437.

Psacharopoulos G. y Ariagada M(1986): "The educational composition of the labor force: An international comparison”, International Labor Review, 125, pp.561-574.

Ray, D.(1998):”Development Economics”, Princeton University Press, U.S.A.

Rebelo, S.(1991): "Long-run Policy Analysis and Long-run Growth", Journal of Political Economy, 99, pp.500-522.

Romer, P.(1986): “Increasing Returns and Long-run Growth”, Journal of Political Economy, 94, pp.1002-1037. , (1986): "Capital Accumulation in the Theory of Long-run Growth", Robert Barro (Ed.), Modern Business Cycle Theory (Cambridge: Harvard University Press), 51-127. , (1990): “Endogenous Technological Change”, Journal of Political Economy, 98, partII. , (1994): “The Origins of Endogenous Growth”, Journal of Economic Perspectives, 8, $\mathrm{N}^{0} 1, \mathrm{pp} .3-22$.

Schultz T.(1961): “Inversión en Capital Humano”. La economía en 1961, Mayor Mayor, P. Madrid.

Solow, R.(1956): “A Contribution to the Theory of Economic Growth”, Quartely Journal of Economics, 70, 1, pp.65-94.

, (1994): "Perspectives on Growth Theory", Journal of Economic Perspectives, 8, 1, pp.45-54.

Stokey, N.(1991): "Human Capital, Product Quality and Growth”, Quartely Journal of Economics, 106, (2), pp.587-616.

Tamura, R., (1995): "Human Capital, Fertility, and Economic Growth.” The Economics of human behavior, Tomassi M and Ierulli, K.Ed. Cambridge.

Topel, R. (1999): "Labor Markets and Economic Growth”. En O.C. Ashenfelter and D.Cards (eds) Handbook of Labor Economics, Vol. 3C, North Holland, Amsterdam.

Utrera,G.(1999): "El Crecimiento Económico en Latinoamérica”, Anales de la Asociación Argentina de Economía Política, Rosario, Argentina.

World Bank (2000): World Development Indicators, CD Rom.

Young A.(1995):, "The tyranny of numbers: confronting the statistical realities of the East Assian Growth experience. Quarterly Journal of Economics, 110(3), pp.641-680. 